\title{
Common and segregated neural substrates for automatic conceptual and affective priming as revealed by event-related functional magnetic resonance imaging
}

\author{
Hongyan Liu ${ }^{a}$, Zhiguo Hu ${ }^{b}$, Danling Peng ${ }^{\mathrm{a}, *}$, Yanhui Yang ${ }^{\mathrm{c}}$, Kuncheng $\mathrm{Li}^{\mathrm{c}}$ \\ a State Key Laboratory of Cognitive Neuroscience and Learning, Beijing Normal University, Beijing, PR China \\ ${ }^{\mathrm{b}}$ Laboratory for Higher Brain Function, Institute of Psychology, Chinese Academy of Sciences, Beijing, PR China \\ ${ }^{\mathrm{c}}$ Xuanwu Hospital, Beijing, PR China
}

\section{A R T I C L E I N F O}

Article history:

Accepted 2 November 2009

Available online 16 December 2009

\section{Keywords:}

Conceptual priming

Affective priming

Lexical decision

Automatic processing

fMRI

\begin{abstract}
A B S T R A C T
The brain activity associated with automatic semantic priming has been extensively studied. Thus far there has been no prior study that directly contrasts the neural mechanisms of semantic and affective priming. The present study employed event-related fMRI to examine the common and distinct neural bases underlying conceptual and affective priming with a lexical decision task. A special type of emotional word, a dual-meaning word containing both conceptual meaning and affective meaning, was adopted as target. Short stimulus onset asynchrony (SOA) $(50 \mathrm{~ms})$ was used to emphasize automatic processing. Fifteen participants were scanned in the present study. We found that the left middle/superior temporal gyrus was the brain region involved in both automatic conceptual and affective priming effects, suggesting general lexical-semantic processing that share in the two types of priming. The left inferior frontal gyrus and right superior temporal gyrus were found to be the conceptual-specific areas in automatic priming effect, consistent with the role of these areas in more extensive within-category semantic processes. The results also revealed that the left fusiform gyrus and left insula were the affective-specific regions in automatic priming effect, demonstrating the involvement of the left fusiform gyrus in automatic affective priming effect, and clarifying the role of the insula in emotional processing rather than conceptual processing. Despite comparable behavioral effects of automatic conceptual priming and affective priming, the present study revealed a neural dissociation of the two types of priming, as well as the shared neural bases.
\end{abstract}

(c) 2009 Elsevier Inc. All rights reserved.

\section{Introduction}

Semantic priming is a typical paradigm used to examine mental representations of word meanings and their relationships (Meyer \& Schvaneveldt, 1971). The semantic priming effect refers to the increased speed and accuracy in recognizing a target preceded by a related prime word (e.g. sheep-cow), compared to an unrelated prime (e.g. table-cow). Semantic priming is thought to reflect the operation of different cognitive processes that make differential contributions depending on experimental conditions and on the semantic relationship between prime and target. For example, when the interval between prime and target (i.e., the stimulus onset asynchrony, SOA) is short, semantic priming is usually explained by an automatic spread of activation through semantic memory (Collins \& Loftus, 1975; Neely, 1991).

The brain activity associated with semantic priming has been extensively studied in normal subjects (e.g., Copland et al., 2003;

\footnotetext{
* Corresponding author. Fax: +86 1058808939

E-mail address: pdl3507@bnu.edu.cn (D. Peng).
}

Gold et al., 2006; Kotz, Cappa, von Cramon, \& Friederici, 2002; Mummery, Shallice, \& Price, 1999; Rissman, Eliassen, \& Blumstein, 2003; Rossell, Bullmore, Williams, \& David, 2001; Rossell, Price, \& Nobre, 2003; Tivarus, Ibinson, Hillier, Schmalbrock, \& Beversdorf, 2006; for a review, see Henson (2003)) and individuals with lesions (e.g., Copland, 2003; Kensinger, Siri, Cappa, \& Corkin, 2003; McDonald et al., 2005; Milberg, Blumstein, \& Dworetzky, 1987; Milberg, Blumstein, Katz, Gershberg, \& Brown, 1995). A large body of literature has implicated several brain regions in semantic priming, such as the left inferolateral frontal areas (Copland et al., 2003; Rossell et al., 2003), anterior cingulate gyrus (Mummery et al., 1999; Rossell et al., 2001), middle/superior temporal cortex (Copland et al., 2003; Gold et al., 2006; Kensinger et al., 2003; Rossell et al., 2001, 2003) and parietal cortex (Rossell et al., 2003).

In addition to semantic priming, another type of priming has been reported. This is the so-called "affective priming", during which a positive or negative prime stimulus (e.g., word or picture) is typically presented for less than $200 \mathrm{~ms}$ and followed by a positive or negative target stimulus after a short interstimulus interval. Results show that the time needed to evaluate the target stimuli as 
either "positive" or "negative" is significantly shorter when prime and target share the same valence (i.e., positive-positive or negative-negative; this is termed "affectively congruent") as compared to trials in which prime and target are of opposite valence (i.e., positive-negative or negative-positive, or "affectively incongruent") (Hermans, De Houwer, \& Eelen, 2001). Thus far the phenomenon of affective priming has been investigated in a number of behavioral studies (e.g., De Houwer, Hermans, Rothermund, \& Wentura, 2002; Fazio, Sanbonmatsu, Powell, \& Kardes, 1986; Hermans, De Houwer, \& Eelen, 1994; Hermans et al., 2001; for a review, see Klauer \& Musch, 2003) and several neuroimaging studies (e.g. Fischer et al., 2003; Luo et al., 2004; Nomura et al., 2004; Wright et al., 2001). The affective priming effect - whereby primes facilitate the semantic encoding of affectively congruent targets - has been consistently observed at short SOAs (for a review, see De Houwer et al., 2002). Neuroimaging studies have found that the primary brain regions involved in subliminal and repetition affective priming were fusiform gyrus and amygdala (e.g., Luo et al., 2004; Nomura et al., 2004; Wright et al., 2001).

A handful of behavioral priming studies have adopted a comparative approach (e.g., De Houwer et al., 2002; Klauer \& Musch, 2002; Klinger, Burton, \& Pitts, 2000; Storbeck \& Robinson, 2004) to compare the two types of priming effect. Significant semantic and affective priming effects have often been demonstrated in the same study. Though the two types of priming have similar reflexes at a behavioral level, whether or not the same underlying mechanisms were implicated in semantic and affective priming at the neural level remains unclear. To our knowledge, no single study has simultaneously investigated the neural basis of semantic priming and affective priming explicitly. Without direct comparison within a single study, the neural mechanisms underlying the two types of priming could be obscured by various other factors. For example, the stimuli used in semantic priming studies are usually neutrally valenced, while stimuli in affective priming studies are often emotionally salient. Moreover, affective priming studies typically adopt tasks that focus on emotional dimensions such as emotional categorization (e.g., judging whether a target is positive or negative). Thus, without an explicitly comparative approach, any conclusions about the common or different neural nature of the two types of priming are potentially problematic.

The present study aims to localize the neural networks - shared and distinct - that underlie both conceptual and affective priming, by using a combined semantic and affective priming paradigm. We employed a particular type of word, which we term "dual-meaning word", was used as target. A dual-meaning word contains two relevant dimensions of information: (i) a conceptual component that reflects an object or action (its "conceptual semantics"), and (ii) a certain attitude or emotive component (its "affective semantics") (Kövecses, 2003). For example, the word '暴君' (oppressor) contains both the concept of '君主' (emperor) and the emotion of '残酷' (cruel). Dual-meaning words are particularly useful for joint examination of semantic and affective priming effects. When a dualmeaning word was primed by a semantically related word, it resulted in a semantic priming pair. Likewise, when it was primed by an affective related word, an affective priming pair was obtained. In our paradigm, we used the same dual-meaning words as the target stimuli for both semantic and affective priming conditions. Additionally, we employed the same behavioral task across both conditions. Thus, any differences between semantic priming and affective priming could be directly assessed without the possibility of being confounded by different targets or tasks across conditions.

Note that affective priming is more of an automatic process (De Houwer et al., 2002; Hermans et al., 1994, 2001; Murphy \& Zajone, 1993), and that reliable affective priming effects were only obtained with shorter SOAs (De Houwer et al., 2002; Fazio et al.,
1986; Hermans et al., 1994, 2001; for a review, see Klauer \& Musch, 2003). Because of these facts, the present study used a short SOA to ensure that affective priming effects would be reliably elicited.

In the priming literature, the lexical decision task (LDT) has been widely used (e.g., Copland et al., 2003; Gold et al., 2006; Kotz et al., 2002; Mummery et al., 1999; Rissman et al., 2003; Rossell et al., 2001, 2003). In this task, participants must decide whether the target item is a real word or not. Due to the lack of a specific task demand (e.g., to evaluate or categorize the target), the LDT does not explicitly favor either semantic or affective priming from a response competition perspective (De Houwer, 2003). Because of this, the LDT should be an appropriate task for measuring spreading activation related to semantic and affective factors (Neely, 1991; Storbeck \& Robinson, 2004). Moreover, a rapid presentation event-related fMRI design (Dale \& Buckner, 1997) was used, which has the advantage of being highly resistant to the subject's response habituation, expectation and strategy compared with blocked and fixed interval event-related fMRI designs, and at the same time it is able to distinguish blood oxygenation level-dependent (BOLD) responses despite signal overlaps (see also Luo et al., 2004).

The goal of this study was to explore the common and distinct neural networks underlying automatic semantic and affective priming. Towards this end, a special type of emotional word, dual-meaning words, were used as target stimuli, and a lexical decision task was adopted. In doing so, the two types of priming could be investigated simultaneously, and compared directly. Based on previous literature, we expected to examine common and specific activities for conceptual and affective priming in the inferior frontal gyrus (IFG), middle temporal gyrus (MTG), superior temporal gyrus (STG), fusiform gyrus (FG), and amygdala. We also wished to examine whether the insula would index semantic or affective priming, in order to gain insight on the debate on the role of the insula in semantic vs. affective processing. Some researchers have argued that the insula is a purely linguistic area used in conceptual processing (e.g., Rossell et al., 2001; Rumsey et al., 1997), while others have suggested that the insula is an area that is responsible for verbal motivation and verbal affect (e.g., Ardila, 1999; Ardila, Benson, \& Flynn, 1997). By improving on the research design, we aimed to provide clearer evidence regarding the degree to which the neural networks involved in semantic and effective priming effects are shared, and the degree to which they are distinct.

\section{Methods}

\subsection{Participants}

Fifteen native Chinese speakers, eight males and seven females, aged from 18 to 25 years, participated in the experiment. They were all right-handed with normal or corrected to normal vision. None had any history of neurological or psychiatric disorders. They all gave informed consent before the experiment.

\subsection{Design}

The study used a typical priming paradigm while adopting a rapid presentation event-related fMRI design (see Fig. 1). The target word was either a dual-meaning word or a non-word. Dual-meaning words were preceded by a prime word that was either related or unrelated with either the target's conceptual or affective meaning. Note that the prime word was not a dual-meaning word, and was either neutrally or negatively valenced. The non-word was also preceded by a prime word. The prime was presented for $50 \mathrm{~ms}$, the offset of which was followed by a target for $1150 \mathrm{~ms}$. 


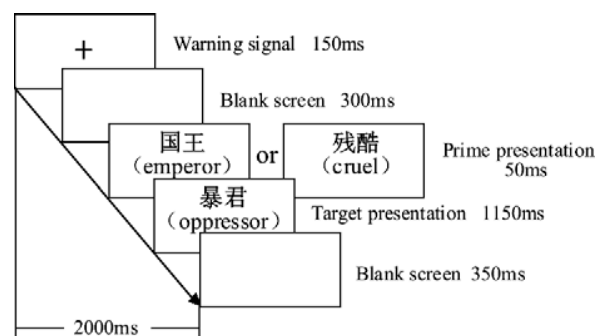

Fig. 1. The priming task. Following a warning signal and a blank screen, a prime word was presented for $50 \mathrm{~ms}$, followed by a target word which was presented for $1150 \mathrm{~ms}$ and another blank screen.

The whole sequence was thus the following: warning signal "+" (150 ms) - blank (300 ms) - prime (50 ms) - target (1150 ms) - blank (350 ms). This constituted one trial corresponding to one TR $(2 \mathrm{~s})$. The baseline was passive viewing with the same sequence, but with the prime and the target both replaced by a blank screen. The baseline was designed in such a way that the occurrence of experimental events and null events appeared totally unexpected to the subject. The subject was required to make a lexical decision on the target word, and respond with his/her right hand if the target was a real word (e.g., “暴君'), and with his/her left hand if the target was a non-word.

As mentioned above, there were four experimental conditions, including conceptual related pairs, conceptual unrelated pairs, affective related pairs and affective unrelated pairs. For each of the four experimental conditions, there were 40 trials. For nonword pairs, there were 160 trials. For null events serving as the baseline, there were 260 trials altogether. Moreover, 40 neutralneutral pairs were added as fillers, which were included to offset the fact that all the real target words were dual-meaning words. All types of trials were randomized. The experiment was performed in four runs counterbalanced across subjects. The two response hands were also counterbalanced across subjects.

\subsection{Stimuli}

A total of 400 Chinese two-character words, 80 negative dualmeaning words, 80 negative words, 240 neutral words, were used in the study for experimental conditions. The emotional valence of these words was rated by 20 additional participants who were not participants of the fMRI experiment. Ratings were based on a 9point Likert scale where 1 represented maximum negative and nine maximum positive valence. The 80 dual-meaning target words were then divided into two separate lists (Lists A and B). Words in Lists A and B were matched for valence (2.31 for List A and 2.54 for List $B, P=0.89$ ). For half of the participants, words in List A were target words for conceptual pairs and List B for affective pairs, while for the other half, words in List B were target words for conceptual pairs and List A for affective pairs. For the conceptual prime word, there was no significant difference in the valence between related and unrelated conditions (5.18 for related neutral prime words and 5.00 for unrelated neutral prime words, $P=0.32$ ). For the affective prime word, there was a significant difference in the valence between related and unrelated conditions ( 2.17 for related negative prime words and 5.04 for unrelated neutral prime words, $P<0.001$ ).

A 5-point scale was used to assess the semantic association between the dual-meaning words and the corresponding conceptual prime words in the conceptual priming pairs, and the affective association between the dual-meaning words and the corresponding affective prime words in the affective priming pairs. Twenty additional participants who were not participants of the fMRI experiment were asked to judge to what extent pairs of words were related. Word pairs with high association were selected, while the two types of association were not significantly different from each other ( 4.15 for the semantic association vs. 4.17 for the affective association, $P=0.72$ ).

The prime words in the non-word pairs were either negatively or neutrally valenced, selected from the same norms and criteria described earlier. The non-word targets consisted of two Chinese characters but with no meaning.

There was no significant difference in the frequency of words $(P>0.05)$, the number of strokes of the characters constituting the words $(P>0.05)$ across all trial types, including conceptual or affective, related or unrelated, for the primes and targets, respectively.

\subsection{Apparatus}

The fMRI experiment was performed on a $1.5 \mathrm{~T}$ Siemens Sonata whole-body MRI scanner. Functional scans were obtained by using a single-shot $\mathrm{T} 2{ }^{*}$-weighted gradient-echo echo planar imaging (EPI) sequence. The following scan parameters were used: TR/TE/ theta $=2000 \mathrm{~ms} / 50 \mathrm{~ms} / 90^{\circ}$, FOV $=230 \times 230 \mathrm{~mm}^{2}$, Matrix $=64 \times$ 64 , and slice thickness $/ g a p=6 \mathrm{~mm} / 1.8 \mathrm{~mm}, 20$ contiguous sagittal slices were acquired to cover the whole brain at 155 time points per run.

\subsection{Experimental procedures}

Before the fMRI scanning, subjects were first familiarized with the experimental procedure, and subsequently they performed the experiment in the scanner. The participant's head was secured by foam rubber to minimize movement.

\section{6. fMRI data analysis}

We used SPM2 (Wellcome Department of Cognitive Neurology, London, UK) implemented in Matlab for image preprocessing and statistical analyzes. Standard preprocessing was conducted comprising slice timing correction, realignment, spatial normalization and smoothing. The functional images were normalized to the EPI template with $2 \times 2 \times 2 \mathrm{~mm}^{3}$ spatial resolution, and then smoothed with a Gaussian filter of $8 \mathrm{~mm}$ full-width at halfmaximum.

A general linear model was used to estimate the condition effect with canonical hrf (hemodynamic response function) for each subject. The data were globally scaled and high-pass-filtered at $128 \mathrm{~s}$. Significant changes in hemodynamic response for each subject and condition were assessed using $t$-test. Individual activation maps of the conceptual and affective priming effects were estimated through contrasts: (conceptually unrelated - conceptually related) and (affectively unrelated - affectively related), respectively. To identify the regions that showed enhancement of activation in the priming, the reverse contrasts (i.e., related - unrelated) were also conducted. The group averaged effects were computed with a random-effects model. For group analyzes, only clusters with more than 10 contiguous voxels activated above the threshold of $P<0.001$ (uncorrected) were considered as significant.

To investigate the common and segregated neural mechanisms for automatic conceptual and affective priming, region of interest (ROI) analyzes were conducted. The regions thought to be involved in semantic and affective priming are selected as ROIs, including the bilateral IFG, MTG, STG, FG, insula and amygdala. First, a cross-priming type overall effect of interest was computed with a random-effect one-way ANOVA model. An inclusive anatomical mask of an ROI (e.g. FG), defined by using the Automated Anatomical Labeling (AAL) template available in the SPM-Toolbox WFU 
PickAtlas (Maldjian, Laurienti, Burdette, \& Kraft, 2003), was used on the overall effect across conceptual and affective priming and then peak activation was computed. The coordinates of the peak activation for the above ROIs were as follows: left IFG $(-26,30$, $-20)$, right IFG $(46,10,14)$, left MTG $(-54,-6,-14)$, right MTG $(44,-66,4)$, left STG $(-54,-6,-12)$, right STG $(62,-10,2)$, left FG $(-38,-20,-22)$, right FG $(42,-22,-20)$, left insula $(-36$, $-10,8)$ and right insula $(38,-10,4)$. An $8 \mathrm{~mm}$ radius sphere ROI was drawn centered on the peak activation voxel in SPM2 using the volume of interest (VOI) toolbar. The amygdala is one of the most important regions in the emotional processing, and it was one of the regions selected a priori for further analysis. Given the small size and the shape of the amygdala, we used the AAL in the WFU PickAtlas to structurally define the amygdala ROI. Small volume correction (SVC, $P<0.05$, false discovery rate (FDR)-corrected) was carried out for these a priori regions of interest (Worsley et al., 1996) in both (conceptually unrelated - related) and (affectively unrelated - related) contrasts. We then extracted the percent signal changes of the peak voxels in the ROI regions that survived after SVC correction by MarsBar toolbox (http://marsbar.sourceforge.net/) for SPM.

\section{Results}

\subsection{Behavioral results}

The mean error rate was $3.8 \%$, which was much lower than chance, indicating that the subject responded to the stimuli in an expected manner. A 2 (priming: unrelated priming/related priming) $\times 2$ (priming type: conceptual/affective) repeated ANOVA on RT (reaction time) indicated a significant main effect of priming $[F(1,14)=12.9, P<0.01]$, but no significant main effect of priming type $[F(1,14)=0.2, P=0.66]$. Post hoc analyzes were performed to see if the priming effect was significant in both priming types. A significant priming effect was obtained in both the concep- tual [M_unrelated $=698.5 \pm 69.5 \mathrm{~ms}$, M_related $=673.7 \pm 60.7 \mathrm{~ms}$; $F(1,14)=33.34, P<0.01]$ and affective [M_unrelated $=698.6 \pm$ $63.3 \mathrm{~ms}$, M_related $=664.9 \pm 52.7 \mathrm{~ms} ; \quad F(1,14)=6.24, \quad P<0.05]$ priming condition. Analyzes showed no interaction between priming (unrelated/related) and priming type (conceptual/affective) $[F(1,14)=0.6, P=0.46]$, suggesting that the amount of priming did not differ significantly between the conceptual and affective priming conditions.

\section{2. fMRI results}

Priming-related BOLD activations for unrelated conditions relative to related conditions, as well as the reverse contrasts, are illustrated in Table 1. The conceptual priming effect resulted from the comparison between pairs where targets preceded by a conceptual unrelated prime word and targets preceded by a conceptual related prime. The conceptual priming effect was associated with decreased activity in several temporal regions, including the left MTG and right STG. Within frontal regions, decreased activation occurred in the left IFG, and the bilateral middle frontal gyrus (MFG). Decreased activity was also observed in the left middle cingulate gyrus and left postcentral gyrus. When contrasted with the affectively unrelated pairs, the affectively related pairs were also associated with decreased activity in several temporal regions, including the left MTG/STG and right STG. Decreased activity was also observed in the left FG, right middle occipital gyrus (MOG), left insula, right insula/putamen and left caudate.

Additionally, other regions also showed a significant enhancement effect when contrasting related pairs with unrelated pairs. The left inferior temporal gyrus (ITG), inferior occipital gyrus (IOG) and MOG showed a conceptual enhancement effect, and the right supplementary motor area (SMA) and left cerebellum showed an affective enhancement effect.

The overall effect across priming types was used to define the ROIs. Six ROIs survived the $P<0.05$ (SVC) threshold, including

Table 1

Regions showing the automatic conceptual and affective priming effects and enhancement effects.

\begin{tabular}{|c|c|c|c|c|c|c|}
\hline \multirow[t]{2}{*}{ Areas } & \multirow[t]{2}{*}{$\mathrm{BA}$} & \multicolumn{3}{|c|}{ MNI coordinates } & \multirow[t]{2}{*}{$Z$ value } & \multirow[t]{2}{*}{ Cluster size } \\
\hline & & $x$ & $y$ & $z$ & & \\
\hline \multicolumn{7}{|l|}{ Unrelated priming vs. related priming } \\
\hline L_inferior/middle frontal gyrus & BA $11 / 47$ & -24 & 32 & -18 & 3.62 & 10 \\
\hline R_middle frontal gyrus & BA 11 & 6 & 44 & -12 & 3.46 & 42 \\
\hline L_middle cingualte gyrus & BA 23 & -6 & -22 & 48 & 3.42 & 13 \\
\hline R_superior temporal gyrus & BA 22 & 66 & -10 & 2 & 3.48 & 14 \\
\hline L_middle temporal gyrus & BA $20 / 21$ & -54 & -8 & -14 & 3.92 & 39 \\
\hline L_postcentral gyrus & BA $3 / 4 / 43$ & -58 & -10 & 38 & 3.52 & 40 \\
\hline \multicolumn{7}{|l|}{ Affective } \\
\hline L_insula & BA 48 & -38 & -12 & 6 & 4.21 & 84 \\
\hline R_insula/putamen & BA 48 & 36 & -12 & 4 & 4.09 & 24 \\
\hline L_superior/middle temporal gyrus & BA $21 / 22 / 41$ & -48 & -34 & 4 & 3.88 & 25 \\
\hline R_superior temporal gyrus & BA 48 & 56 & -6 & 5 & 3.59 & 24 \\
\hline L_fusiform gyrus & BA 37 & -34 & -40 & -22 & 3.47 & 22 \\
\hline R_middle occipital gyrus & BA 19 & 34 & -66 & 28 & 3.87 & 29 \\
\hline L_caudate & & -6 & 6 & 14 & 3.60 & 22 \\
\hline \multicolumn{7}{|l|}{ Related priming vs. unrelated priming } \\
\hline L_inferior temporal gyrus & BA 20 & -42 & -24 & -22 & 4.14 & 16 \\
\hline L_inferior occipital gyrus & BA 18 & -26 & -92 & -4 & 3.66 & 74 \\
\hline L_middle occipital gyrus & BA 18 & -24 & -86 & 4 & 3.57 & \\
\hline \multicolumn{7}{|l|}{ Affective } \\
\hline R_supplementary motor area & BA 6 & 6 & 10 & 60 & 3.98 & 16 \\
\hline L_cerebellum & & -20 & -72 & -20 & 3.58 & 12 \\
\hline L_cerebellum & & -34 & -64 & -28 & 3.31 & 17 \\
\hline
\end{tabular}

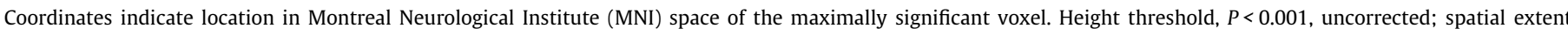
threshold, 10 voxels. Abbreviations: L, left hemisphere; R, right hemisphere; BA, Brodmann's area. 
the left IFG, left MTG/STG, right STG, left FG, left insula and right insula. No significant activation was found in the structurally defined amygdala at $P<0.05$ SVC. A within-subject two-way ANOVA was used to examine the priming (unrelated and related priming) and priming type (conceptual and affective priming) effects for the above six ROIs based on percent signal change for each condition. Main effects and interactions for these ANOVAs are reported, as well as post hoc analyzes (see Fig. 2).

There was significant main effect of priming in the left MTG/STG $[F(1,14)=35.52, P<0.001]$, but no significant main effect of priming type $[F(1,14)=0.15, P=0.70]$ and no significant interaction effects $[F(1,14)=0.007, P=0.93]$ in this region. Post hoc analyzes confirmed that the left MTG/STG showed greater activation in unrelated than related pairs in both conceptual $[F(1,14)=22.72$, $P<0.001]$ and affective $[F(1,14)=11.32, P<0.01]$ conditions.

There were significant interaction effects in the left IFG $[F(1,14)=6.22, P<0.05]$, left FG $[F(1,14)=14.28, P<0.01]$, left insula $[F(1,14)=10.08, P<0.01]$ and right insula $[F(1,14)=5.34$, $P<0.05]$. While in the right STG, the interaction effect was only marginally significant $[F(1,14)=4.13, P=0.062]$. Post hoc analyzes showed that there were significant conceptual priming effects in the left IFG $[F(1,14)=16.83, P<0.001]$ and the right STG $[F(1,14)=15.93, P<0.001]$, and significant affective priming effects in the left FG $[F(1,14)=16.41, P<0.001]$, left insula $[F(1,14)=27.22$, $P<0.001]$ and right insula $[F(1,14)=14.46, P<0.01]$. There were no other priming effects in these regions. The data indicated that the activities of the right insula in the related and unrelated conditions were weaker than the baseline (see also Fig. 2C), and as such, this area is not further discussed.

\section{Discussion}

The present study examined the neural correlates of automatic conceptual and affective priming simultaneously using event-related fMRI with a lexical decision task. We are unaware of any prior fMRI or PET study that has directly explored the commonalities and differences between semantic and affective priming effects in the brain. Our study thus represents the first attempt at addressing this issue.

\subsection{Behavioral priming}

Behavioral results indicated a significant priming effect in both the conceptual and affective conditions, with facilitation of the conceptually/affectively related condition relative to the unrelated condition. Such findings are consistent with other studies exploring automatic semantic priming (Copland et al., 2003; Gold et al., 2006; Kotz et al., 2002; Mummery et al., 1999; Rossell et al., 2001, 2003) and affective priming (De Houwer et al., 2002; Fazio et al., 1986; Hermans et al., 1994, 2001; Luo et al., 2004; for a review, see Klauer \& Musch, 2003). The replication of the conceptual and affective priming effects in our fMRI experiment confirms that the subjects' behavior and task performance were normal during scanning. The results also revealed that, at the behavioral level, the priming effect did not differ significantly between the conceptual and affective priming conditions.

\subsection{Common brain regions involved in automatic conceptual and affective priming effect}

The present study showed that the left MTG/STG exhibited both significant conceptual and affective priming effects. The amount of priming in this region did not differ significantly between the two types of priming (see Fig. 2A). As conceptual semantics and affective semantics reflect two aspects of semantic information, they may share common neural representations of semantics. The left STG is normally associated with reduced activation in semantic priming (e.g., Mummery et al., 1999; Rissman et al., 2003). This may reflect differential modulation resulting from the automatic activation of shared semantic representations (Rissman et al., 2003). It may also be the case that the role of the STG in semantic processing is to serve as a relay to and from other temporal lobe structures (Rissman et al., 2003). The left MTG was found to be involved in both automatic conceptual and affective priming in the
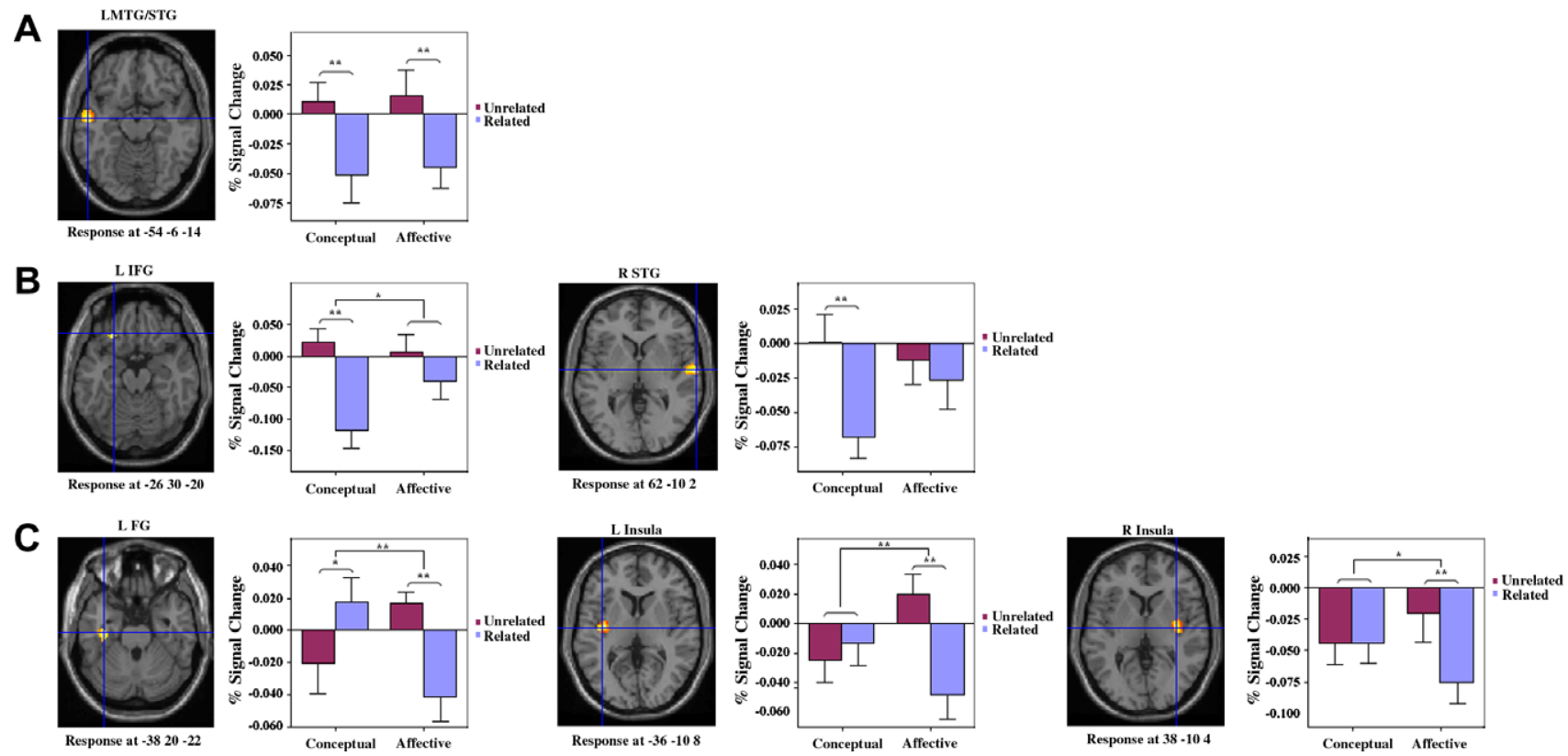

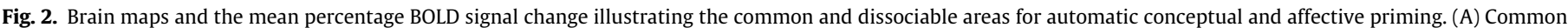

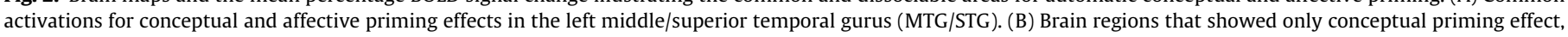

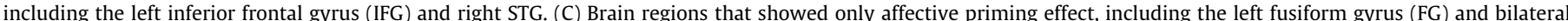

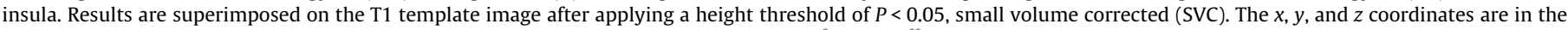
MNI space. The error bars show standard errors. L, left hemisphere; R, right hemisphere. ${ }^{*} P<0.05,{ }^{* * *} P<0.01$. 
present study, which is also consistent with a large body of literature that implicates this region in semantic processing. Many neuroimaging studies have noted the contribution of the temporal cortex to semantic memory and semantic processing (Copland et al., 2003; Mummery et al., 1999; Rissman et al., 2003; Rossell et al., 2001). In particular, neuroimaging studies have implicated the left middle temporal area in a range of lexical-semantic operations including semantic priming, lexical decision, and semantic judgment (e.g., Binder et al., 1997; Booth et al., 2002; Copland et al., 2003; Friederici, Opitz, \& Cramon, 2000; Price, Moore, Humphreys, \& Wise, 1997; Rossell et al., 2001), and the involvement in semantic processing of the MTG is modality independent (Liu et al., 2009). The role of the left MTG/STG in semantic processing is also consistent with the clinical literature (Hodges, Patterson, Oxbury, \& Funnell, 1992; Martin \& Chao, 2001). The common reduced activity in the left MTG/STG in both the conceptual and affective priming suggests that the perception of the prime word activates a lexical-semantic network that shares common elements with the target word, and so the target can be recognized with greater neural efficiency (Rissman et al., 2003).

An alternative explanation for the common implication of the left MTG/STG in both conceptual and affective priming is that this region contributes to both semantic processing and emotional processing. In fact, there exist neuroimaging results that suggest that the left MTG is also implicated in the processing of emotional information, such as emotional pictures and emotional tone (e.g., Lane, Fink, Chau, \& Dolan, 1997; Morris et al., 1996). Current evidence also suggests that the STG is engaged in emotional perception and processing (Adolphs, 2002; Meyer, Alter, Friederici, Lohmann, \& von Cramon, 2002; Mitchell, Elliott, Barry, Cruttenden, \& Woodruff, 2003).

\subsection{Conceptual-specific neural activities in automatic priming effect}

We found that the left IFG and right STG (BA 22) are areas that are selectively involved in automatic conceptual priming, but not in affective priming.

The involvement of the left IFG in conceptual priming is in accordance with the role of this region in semantic processing. The left prefrontal cortex plays an important role in semantic priming due to its proposed role in the retrieval of semantic information (e.g., Booth et al., 2002; Buckner, Koutstaal, Schacter, \& Rosen, 2000; Buckner et al., 1995; Kotz et al., 2002; McDonald et al., 2005). This finding is consistent with other similar studies. A number of priming studies have shown that the activity of this region is attenuated by priming (e.g., Buckner et al., 1995, 2000; Copland et al., 2003; Donaldson, Petersen, \& Buckner, 2001; Kotz et al., 2002; Wagner, Koutstaal, Maril, Schacter, \& Buckner, 2000). For example, an fMRI study by Kotz et al. (2002) found that the contrast between unrelated and related target words was associated with increased activation of the left IFG, suggesting that the suppression effect in the left IFG is due to reduced demand for semantic memory retrieval or the result of selection caused by the automatic semantic priming effect. Copland et al. (2003) also found reduced activity in the ventral anterior portion of the left inferior prefrontal cortex for conceptual priming. Reduced activity in the left IFG has also been found to be associated with conceptual repetition priming (Wagner et al., 2000). Furthermore, there is lesion data implicating the inferolateral frontal region in semantic priming, which suggests an automatic role in semantic processing (e.g., Milberg et al., 1987, 1995). Our result adds to this body of neuroimaging studies identifying the left IFG as being critical to semantic operations.

The present study also showed that the right STG was involved in conceptual priming. This is in accordance with other similar studies. For example, Rossell et al. (2001) found activation of the right STG during semantic priming tasks at short and long SOA.
There are an increasing number of studies that relate right STG activation to various aspects of semantics, which presumably reflects a component of the response to correct matching (Rossell et al., 2001). For example, Kotz, von Cramon, and Friederici (1999) noted right STG activation during semantic categorization. In our study, the conceptual prime word and target dual-meaning word have some shared semantic components (e.g., the prime word '君主' (emperor) and the target word “暴君' (oppressor) has both the concept of person and emperor), while the affective prime word and target word did not share the same semantic category, instead having only emotional association (e.g., the prime word '残酷' (cruel) and the target word “暴君' (oppressor)). Thus the lateralization in the fMRI data may reflect the fact that the right STG is involved in broader, more extensive within-category semantic processes (Rossell et al., 2001).

\subsection{Affective-specific neural activities in automatic priming effect}

We also found that the left FG and left insula are areas that are selectively involved in automatic affective priming, but not in conceptual priming.

The fusiform gyrus is an area that has been shown to be sensitive to emotional manipulation. Many studies have demonstrated that activity in the FG is modulated by the emotional valence of materials. For example, bilateral activation in the fusiform gyrus was found during the viewing (e.g., Lane et al., 1997) and evaluation of unpleasant pictures (e.g., Paradiso et al., 1999). In addition, a number of studies have demonstrated that the priming effect in the brain is modulated by emotional valence, and that the occipitotemporal visual cortex is particularly sensitive to this (e.g., Rotshtein, Malach, Hadar, Graif, \& Hendler, 2001; Sugase, Yamane, Ueno, \& Kawano, 1999). In these studies, however, the stimuli were presented at a conscious level. To investigate if the emotionality of stimuli modulates the subliminal priming effect in the left FG, Luo et al. (2004) conducted a subliminal repetition priming study, in which the subjects were asked to make a perceptual judgment on the target (i.e., upright/italic judgment). They found that the left fusiform gyrus was associated with the priming effect related to both positive and negative words, but not neural words. Unlike Luo et al., however, we adopted an automatic priming paradigm and a LDT task requiring relatively deep semantic processing to address the conceptual/affective association between prime and target. This had the effect of highlighting the role of the left fusiform gyrus in automatic affective priming effect.

There is a longstanding debate on the role of the insula in semantic and affective processing. While some researchers have argued that the insula is a purely linguistic area used in conceptual processing (Rossell et al., 2001; Rumsey et al., 1997), others have instead suggested that the insula is an area that is important for verbal motivation and verbal affect (Ardila, 1999; Ardila et al., 1997). While the role of the insula is not yet clear, the joint investigation of conceptual priming and affective priming in the present study provides a good chance to shed some light on this particular question. Our results revealed that the insula was implicated in the affective priming effect, but not in the conceptual priming effect (see also Fig. 2C), lending support to theories that emphasizes the role of the insula in affective processing. This finding is consistent with previous studies regarding the role of the insula in processing of unpleasant stimuli, such as the detection of disgust faces (e.g., Phillips et al., 1998), perception of pain (e.g., Treede, Apkarian, Bromm, Greenspan, \& Frederich, 1999), and analysis of distressing stimuli (e.g., Reiman et al., 1997). Therefore, our study helps to clarify the role of the insula by noting the dissociation of conceptual and affective priming effects in this area.

Our results also showed that the comparison of affectively unrelated and related pairs did not evoke significant activation in the 
amygdala, which is a crucial brain region for emotion processing (for reviews, see Dalgleish, 2004; Phillips, Drevets, Rauch, \& Lane, 2003). The lack of activation of the amygdala in automatic affective priming is consistent with Luo et al. (2004)'s results, but is inconsistent with other results (e.g. Fischer et al., 2003; Nomura et al., 2004; Wright et al., 2001). In both the present and Luo et al.'s studies, emotional words were employed, while in other studies (e.g. Fischer et al., 2003; Nomura et al., 2004; Wright et al., 2001), emotional (i.e., fearful or angry) facial expressions were used. As emotional words generally have lower emotional arousal than emotional faces, it is possible that the amygdala was not activated in these two studies due to the low arousal level of the emotional words. Another possibility is that activation of the amygdala might require some amount of attention that is oriented to the emotional dimension of the stimulus (see Pessoa, McKenna, Gutierrez, \& Ungerleider, 2002). If this were the case, the non-emotional tasks used in the present study (i.e., LDT) and Luo et al.'s (i.e., perceptual judgment) may fail to activate the amygdala.

\subsection{Other areas showing the automatic enhancement effect}

In addition to the areas that showed a suppression effect (i.e., the reduced neural responses associated with related relative to unrelated pairs), there were other areas that exhibited an enhancement effect, in which there was increased activity in related pairs than unrelated pairs. For automatic conceptual priming, enhancement effects were found in the left ITG, IOG and MOG. This was consistent with the role of the left lateral and inferior temporal cortices in explicit lexical-semantic decision (Gold et al., 2006) and with findings on the role of the occipital lobe in the processing of Chinese at both attentional and non-attentional levels (Peng et al., 2003; Tan et al., 2000). For automatic affective priming, the right SMA and left cerebellum showed enhancement effects. Previous studies have shown that emotional stimuli lead to greater physiological responses than neutral ones do (e.g., Lang et al., 1998; Luo et al., 2004). Such physiological responses might have been evoked even at the automatic level, leading to an increased activation in the SMA and cerebellum.

\section{Conclusions}

In summary, by using a combined semantic and affective priming paradigm and dual-meaning words as target, the present study identified the common and distinct cerebral substrates for automatic conceptual and affective priming effects. We found that the left MTG/STG was the common brain region involved in automatic conceptual and affective priming effects, suggesting a general lexical-semantic processing that is shared by the two types of priming. The left IFG and right STG were shown to be conceptual-specific areas in the automatic priming effect, consistent with the role of these areas in more extensive within-category semantic processes. The results also revealed that the left FG and left insula were affective-specific regions in the automatic priming effect, demonstrating the involvement of the left FG in automatic affective priming effect, and clarifying the role of the insula in emotional rather than semantic processing. Despite the comparable automatic conceptual priming effect and affective priming effect at the behavioral level, the present study revealed a neural dissociation of the two types of priming, as well as a shared neural basis.

\section{Acknowledgments}

This work was supported by the National Natural Science Foundation of China (Grant Nos. 30670705 and 30700234) and Program for Changjiang Scholars and Innovative Research Team in Univer- sity (PCSIRT). We thank two reviewers for their valuable comments for previous version of the manuscript, and Brian Dillon for his proofreading of the manuscript.

\section{References}

Adolphs, R. (2002). Neural systems for recognizing emotion. Current Opinion in Neurobiology, 12, 169-177.

Ardila, A. (1999). The role of insula in language: An unsettled question. Aphasiology, $13,79-87$.

Ardila, A., Benson, D. F., \& Flynn, F. G. (1997). Participation of the insula in language. Aphasiology, 11, 1159-1169.

Binder, J. B., Frost, J. A., Hammeke, T. A., Cox, R. W., Bao, S. M., \& Prieto, T. (1997). Human brain language areas identified by functional magnetic resonance imaging. The Journal of Neuroscience, 17(1), 353-362.

Booth, J. R., Burman, D. D., Meyer, J. R., Gitelman, D. R., Parrish, T. B., \& Mesulam, M. M. (2002). Modality independence of word comprehension. Human Brain Mapping, 16, 251-261.

Buckner, R. L., Koutstaal, W., Schacter, D. L., \& Rosen, B. R. (2000). Functional MRI evidence for a role of frontal and inferior temporal cortex in amodal components of priming. Brain, 123, 620-640.

Buckner, R. L., Petersen, S. E., Ojemann, J. G., Miezin, F. M., Squire, L. R., \& Raichle, M E. (1995). Functional anatomical studies of explicit and implicit memory retrieval tasks. The Journal of Neuroscience, 15, 12-29.

Collins, A. M., \& Loftus, E. F. (1975). A spreading-activation theory of semantic processing. Psychological Review, 82, 407-428.

Copland, D. A. (2003). The basal ganglia and semantic engagement: Potential insights from semantic priming in individuals with subcortical vascular lesions, Parkinson's disease, and cortical lesions. Journal of the International Neuropsychological Society, 9, 1041-1052.

Copland, D. A., de Zubicaray, G. I., McMahon, K., Wilson, S. J., Eastburn, M., \& Chenery, H. J. (2003). Brain activity during automatic semantic priming revealed by event-related functional magnetic resonance imaging. NeuroImage, 20, 302-310.

Dale, A. M., \& Buckner, R. L. (1997). Selective averaging of rapidly presented individual trials using fMRI. Human Brain Mapping, 5, 329-340.

Dalgleish, T. (2004). The emotional brain. Nature Reviews Neuroscience, 5, 582-589.

De Houwer, J. (2003). A structural analysis of indirect measures of attitudes. In J. Musch \& K. C. Klauer (Eds.), The psychology of evaluation: Affective processes in cognition and emotion (pp. 219-244). Mahwah, NJ: Lawrence Erlbaum.

De Houwer, J., Hermans, D., Rothermund, K., \& Wentura, D. (2002). Affective priming of semantic categorisation responses. Cognition and Emotion, 16, 643-666.

Donaldson, D. I., Petersen, S. E., \& Buckner, R. L. (2001). Dissociating memory retrieval processes using fMRI: Evidence that priming does not support recognition memory. Neuron, 31, 1047-1059.

Fazio, R. H., Sanbonmatsu, D. M., Powell, M. C., \& Kardes, F. R. (1986). On the automatic activation of attitudes. Journal of Personality and Social Psychology, 50, 229-238.

Fischer, H., Wright, C. I., Whalen, P. J., McInerney, S. C., Shin, L. M., \& Rauch, S. L. (2003). Brain habituation during repeated exposure to fearful and neutral faces: A functional MRI study. Brain Research Bulletin, 59, 387-392.

Friederici, A. D., Opitz, B., \& Cramon, D. Y. (2000). Segregating semantic and syntactic aspects of processing in the human brain: An fMRI investigation of different word types. Cerebral Cortex, 10, 698-705.

Gold, B. T., Balota, D. A., Jones, S. J., Powell, D. K., Smith, C. D., \& Anderson, A. D. (2006). Dissociation of automatic and strategic lexical-semantics: Functional magnetic resonance imaging evidence for differing roles of multiple frontotemporal regions. The Journal of Neuroscience, 26, 6523-6532.

Henson, R. N. A. (2003). Neuroimaging studies of priming. Progress in Neurobiology, $70,3-81$.

Hermans, D., De Houwer, J., \& Eelen, P. (1994). The affective priming effect: Automatic activation of evaluative information in memory. Cognition and Emotion, 8, 515-533.

Hermans, D., De Houwer, J., \& Eelen, P. (2001). A time course analysis of the affective priming effect. Cognition and Emotion, 15, 143-165.

Hodges, J., Patterson, K., Oxbury, S., \& Funnell, E. (1992). Semantic dementia: Progressive fluent aphasia with temporal lobe atrophy. Brain, 115, 1783-1806.

Kensinger, E. A., Siri, S., Cappa, S. F., \& Corkin, S. (2003). Role of the anterior temporal lobe in repetition and semantic priming: Evidence from a patient with a category-specific deficit. Neuropsychologia, 41, 71-84.

Klauer, K., \& Musch, J. (2002). Goal-dependent and goal-independent effects of irrelevant evaluations. Personality and Social Psychology Bulletin, 28, 802-814.

Klauer, K., \& Musch, J. (2003). Affective priming: Findings and theories. In J. Musch \& K. C. Klauer (Eds.), The psychology of evaluation: Affective processes in cognition and emotion (pp. 7-49). Mahwah, NJ: Lawrence Erlbaum.

Klinger, M., Burton, P., \& Pitts, S. (2000). Mechanisms of unconscious priming: I. Response competition, not spreading activation. Journal of Experimental Psychology: Learning, Memory, and Cognition, 26, 441-455.

Kotz, S. A., Cappa, S. F., von Cramon, D. Y., \& Friederici, A. D. (2002). Modulation of the lexical-semantic network by auditory semantic priming: An event-related functional MRI study. NeuroImage, 17, 1761-1772.

Kotz, S. A., von Cramon, D. Y., \& Friederici, A. D. (1999). Priming as a function of semantic information types: an fMRI investigation. NeuroImage, 9, S1000. 
Kövecses, Z. (2003). Metaphor and emotion: Language, culture, and body in human feeling. Cambridge: Cambridge University Press.

Lane, R. D., Fink, G. R., Chau, P. M., \& Dolan, R. J. (1997). Neural activation during selective attention to subjective emotional responses. NeuroReport, 8, 3969-3972.

Lang, P. J., Bradley, M. M., Fitzsimmons, J. R., Cuthbert, B. N., Scott, J. D., \& Moulder, B. (1998). Emotional arousal and activation of the visual cortex: An fMRI analysis. Psychophysiology, 35, 1-13.

Liu, L., Deng, X., Peng, D., Cao, F., Ding, G., Jin, Z., et al. (2009). Modality- and taskspecific brain regions involved in Chinese lexical processing. Journal of Cognitive Neuroscience, 21, 1473-1487.

Luo, Q., Peng, D. L., Jin, Z., Xu, D., Xiao, L. H., \& Ding, G. S. (2004). Emotional valence of word modulates the subliminal repetition priming effect in the left fusiform gyrus: An event-related fMRI study. Neurolmage, 21, 414-421.

Maldjian, J. A., Laurienti, P. J., Burdette, J. B., \& Kraft, R. A. (2003). An automated method for neuroanatomic and cytoarchitectonic atlas-based interrogation of fMRI data sets. Neurolmage, 19, 1233-1239.

Martin, A., \& Chao, L. L. (2001). Semantic memory and the brain: Structure and processes. Current Opinion in Neurobiology, 11, 194-201.

McDonald, C. R., Bauer, R. M., Filoteo, J. V., Grande, L., Roper, S. N., Buchanan, R. J., et al. (2005). Semantic priming in patients with right frontal lobe lesions. Journal of the International Neuropsychological Society, 11, 132-143.

Meyer, M., Alter, K., Friederici, A. D., Lohmann, G., \& von Cramon, D. Y. (2002). fMRI reveals brain regions mediating slow prosodic modulations in spoken sentences. Human Brain Mappng, 17, 73-88.

Meyer, D. E., \& Schvaneveldt, R. W. (1971). Facilitation in recognition pairs of words: Evidence of a dependence between retrieval operations. Journal of Experimental Psychology: General, 90, 227-234.

Milberg, W., Blumstein, S. E., \& Dworetzky, B. (1987). Processing of lexical ambiguities in aphasia. Brain and Language, 31, 138-150.

Milberg, W., Blumstein, S. E., Katz, D., Gershberg, F., \& Brown, T. (1995). Semantic facilitation in aphasia: Effects of time and expectancy. Journal of Cognitive Neuroscience, 7, 33-50.

Mitchell, R. I., Elliott, R., Barry, M., Cruttenden, A., \& Woodruff, P. W. R. (2003). The neural response to emotional prosody, as revealed by functional magnetic resonance imaging. Neuropsychologica, 4, 1410-1421.

Morris, J. S., Frith, C. D., Perrett, D. I., Rowland, D., Young, A. W., Calder, A. J., et al. (1996). A differential neural response in the human amygdala to fearful and happy facial expressions. Nature, 383, 812-815.

Mummery, C. J., Shallice, T., \& Price, C. J. (1999). Dual-process model in semantic priming: A functional imaging perspective. Neurolmage, 9, 516-525.

Murphy, S. T., \& Zajone, R. B. (1993). Affect, cognition, and awareness: Affective priming with optimal and suboptimal stimulus exposures. Jouranl of Personality and Social Psychology, 64, 723-739.

Neely, J. H. (1991). Semantic priming effects in visual word recognition: A selective review of current findings and theories. In D. Besner \& G. W. Humphreys (Eds.), Basic processes in reading: Visual word recognition (pp. 264-336). Erlbaum: New Jersy.

Nomura, M., Ohira, H., Haneda, K., Iidaka, T., Sadato, N., Okada, T., et al. (2004). Functional association of the amygdala and ventral prefrontal cortex during cognitive evaluation of facial expressions primed by masked angry faces: An event-related fMRI study. Neurolmage, 21, 352-363.

Paradiso, S., Johnson, D. L., Andreasen, N. C., O'Leary, D. S., Watkins, G. L., Ponto, L. L., et al. (1999). Cerebral blood flow changes associated with attribution of emotional valence to pleasant, unpleasant, and neutral visual stimuli in a PET study of normal subjects. The American Journal of Psychiatry, 156, 1618-1629.
Peng, D. L., Xu, D., Jin, Z., Luo, Q., Ding, G. S., Perry, C., et al. (2003). Neural basis of the non-attentional processing of briefly presented words. Human Brain Mapping $18,215-221$.

Pessoa, L., McKenna, M., Gutierrez, E., \& Ungerleider, L. G. (2002). Neural processing of emotional faces requires attention. The Proceedings of the National Academy of Science, 99, 11458-11463.

Phillips, M. L., Drevets, W. C., Rauch, S. L., \& Lane, R. (2003). Neurobiology of emotion perception I: The neural basis of normal emotion perception. Biological Psychiatry, 54, 504-514.

Phillips, M. L., Young, A. W., Scott, S. K., Calder, A. J., Andrew, C., Giampietro, V., et al (1998). Neural response to facial and vocal expressions of fear and disgust. Proceedings of the Royal Society of London. Biological Sciences, 265, 1809-1817.

Price, C. J., Moore, C. J., Humphreys, G. W., \& Wise, R. J. S. (1997). Segregating semantic from phonological processes during reading. Journal of Cognitive Neuroscience, 9, 727-733.

Reiman, E. M., Lane, R. D., Ahern, G. L., Schwartz, G. E., Davidson, R. J., Friston, K. J., et al. (1997). Neuroanatomical correlates of externally and internally generated human emotion. The American Journal of Psychiatry, 154, 918-925.

Rissman, J., Eliassen, J. C., \& Blumstein, S. E. (2003). An event-related fMR investigation of implicit semantic priming. Journal of Cognitive Neuroscience, 15, 1160-1175.

Rossell, S. L., Bullmore, E. T., Williams, S. C. R., \& David, A. S. (2001). Brain activation during automatic and controlled processing of semantic relations: A priming experiment using lexical decision. Neuropsychologia, 39, 1167-1176.

Rossell, S. L., Price, C. J., \& Nobre, A. C. (2003). The anatomy and time course of semantic priming investigated by fMRI and ERPs. Neuropsychologia, 41 550-564.

Rotshtein, P., Malach, R., Hadar, U., Graif, M., \& Hendler, T. (2001). Feeling or features: Different sensitivity to emotion in high-order visual cortex and amygdale. Neuron, 32, 747-757.

Rumsey, J. M., Horwitz, B., Donohue, B. C., Nace, K., Maisog, J. M., \& Andreason, P. (1997). Phonological and orthographic components of word recognition: A PETrCBF study. Brain, 120, 739-759.

Storbeck, J., \& Robinson, M. D. (2004). Preferences and inferences in encoding visual objects: A systematic comparison of semantic and affective priming. Personality and Social Psychology Bulletin, 30, 81-93.

Sugase, Y., Yamane, S., Ueno, S., \& Kawano, K. (1999). Global and fine information coded by single neurons in the temporal visual cortex. Nature, 400, 869873.

Tan, L. H., Spinks, J. A., Gao, J. H., Liu, H. L., Perfetti, C. A., Xiong, J., et al. (2000). Brain activation in the processing of Chinese characters and words: A functional MRI study. Human Brain Mapping, 10, 16-27.

Tivarus, M. E., Ibinson, J. W., Hillier, A., Schmalbrock, P., \& Beversdorf, D. Q. (2006). An fMRI study of semantic priming: Modulation of brain activity by varying semantic distances. Cognitive and Behavioral Neurology, 19, 194-201.

Treede, R. D., Apkarian, A. V., Bromm, B., Greenspan, J. D., \& Frederich, A. L. (1999) The cortical representation of pain. Pain, 79, 105-111.

Wagner, A. D., Koutstaal, W., Maril, A., Schacter, D. L., \& Buckner, R. L. (2000). Taskspecific repetition priming in the left inferior prefrontal cortex. Cerebral Cortex, $10,1176-1184$

Worsley, K. J., Marrett, S., Neelin, P., Vandal, A. C., Friston, K. J., \& Evans, A. C. (1996) A unified statistical approach for determining significant signals in images of cerebral activation. Human Brain Mapping, 4, 58-73.

Wright, C. I., Fischer, H., Whalen, P. J., Mclnerney, S. C., Shin, L. M. \& Rauch, S. L. (2001). Differential prefrontal cortex and amygdale habituation to repeatedly presented emotional stimuli. NeuroReport, 12, 379-383. 